\title{
Characterization of sub-two-cycle pulses from a hollow-core fiber compressor in the spatiotemporal and spatiospectral domains
}

\author{
Benjamín Alonso $\cdot$ Miguel Miranda $\cdot$ Francisco Silva $\cdot$ \\ Vladimir Pervak · Jens Rauschenberger • Julio San Román • \\ Íñigo J. Sola $\cdot$ Helder Crespo
}

Received: 20 December 2012/Accepted: 7 March 2013/Published online: 27 March 2013

(C) Springer-Verlag Berlin Heidelberg 2013

\begin{abstract}
We have post-compressed 25 fs (Fourier limit) amplified pulses in an argon-filled hollow-core fiber. The output pulses were compressed using a pair of wedges and chirped mirrors down to $4.5 \mathrm{fs}$ (Fourier limit of $4.1 \mathrm{fs}$ ), which corresponds to less than two optical cycles. We then performed the characterization of the pulses by combining the d-scan and the STARFISH techniques. The temporal (and spectral) measurement of the pulses is done with d-scan, which is used as the reference to extend the characterization to the spatiotemporal (and spatiospectral) amplitude and phase of the pulses by means of STARFISH. The post-compressed pulses at the output of the hollowfiber had an energy of $150 \mu \mathrm{J}$. The analysis of the pulses revealed larger spectral broadening and blue-shift, and shorter duration at the center of the beam. For the first time, we demonstrate the complete characterization of intense
\end{abstract}

B. Alonso $(\varangle)$ · J. San Román · Í. J. Sola

Grupo de Investigación en Óptica Extrema (GIOE), Universidad

de Salamanca, Pl. de la Merced s/n, 37008 Salamanca, Spain

e-mail: b.alonso@usal.es

M. Miranda $\cdot$ F. Silva $\cdot$ H. Crespo

IFIMUP-IN and Departamento de Física e Astronomia,

Universidade do Porto, Rua do Campo Alegre 687,

4169-007 Porto, Portugal

M. Miranda

Department of Physics, Lund University,

P.O. Box 118, SE-221 00, Lund, Sweden

V. Pervak $\cdot$ J. Rauschenberger

UltraFast Innovations $\mathrm{GmbH}$, Am Coulombwall 1,

85748 Garching, Germany

V. Pervak

Department für Physik, Ludwig-Maximilians-Universität

München, Am Coulombwall 1, 85748 Garching, Germany ultra-broadband pulses in the sub-two-cycle regime, which provides an improved insight into the properties (space-time and space-frequency) of the pulses and is highly relevant for their applications.

\section{Introduction}

The advent of sources capable of delivering ultrashort and ultra-intense light pulses has led to numerous applications in atomic, molecular and nuclear physics [1-3]. In particular, intense few-cycle pulses have opened the way for attosecond physics [4] and metrology [5] via the extreme ultraviolet (XUV) attosecond pulse trains that can be obtained by high-harmonic generation (HHG) [6]. Intense near-infrared pulses close to the single-cycle regime have made possible the generation of isolated attosecond pulses $[5,7,8]$.

The technique of chirped pulse amplification (CPA) combined with Ti:sapphire laser technology has provided many laboratories with intense ultrashort pulses in the 20-100 fs range (the lower limit essentially imposed by gain narrowing effects). Although sub-10-fs pulses can be directly obtained from CPA [9] and optical parametric CPA systems [10], they have proven challenging and are still the subject of much research and development. For this reason, two post-compression techniques are usually employed for the generation of intense few-cycle pulses, based on the spectral broadening of light either during propagation in a gas-filled hollow-core fiber (HCF) [11, 12] or during the self-guiding due to the filamentation of light [13]. The nonlinear nature of the spectral broadening process, originating mainly from self-phase modulation (SPM), provides a broader spectrum in the center of the beam where the intensity of the pulse is higher. As a 
consequence, the post-compressed pulses are inhomogeneous and present spatial chirp. The temporal profile of filament-compressed pulses has been shown to depend on the radial coordinate [14]. Also, many efforts are being devoted to the characterization of the filament propagation in terms of temporal [15], spatiotemporal [16, 17] and peak intensity dynamics [18]. The spatial chirp after filamentation and HCF post-compression has been compared experimentally through analysis of the corresponding spectral contents, with more even and less chirped spectra being reported for the HCF case [19]. Recently, a spatially resolved measurement of the spectral and temporal profile of the output mode of an HCF has been performed [20].

Over the last decades, different optical techniques have been introduced for the temporal characterization of ultrashort laser pulses [21]. Most of these now "standard" techniques have been adapted for the temporal measurement of ultra-broadband few-cycle pulses [20, 22-24]. The waveform of sub-4-fs pulses has also been extracted from attosecond streaking [20, 25]. Very recently, the new technique of d-scan (dispersion scan) was introduced, enabling the simultaneous compression and temporal characterization of few-cycle pulses [26, 27]. However, solely temporal characterization of the pulse in a small section of the beam, without accounting for possible variations across the whole pulse-front, is generally insufficient, due to the spatiotemporal coupling effects typical of the nonlinear phenomena employed in the postcompression process. For this purpose, the technique of STARFISH (SpatioTemporal Amplitude-and-phase Reconstruction by Fourier-transform of Interference Spectra of Highly-complex-beams) was proposed [28]. Very recently, STARFISH has been demonstrated with sub-8-fs pulses delivered by an ultrafast oscillator, and its capabilities for the study of ultra-broadband pulses have been analyzed [29].

In this work, we generated and characterized sub-twocycle 4.5 fs pulses (4.1 fs Fourier-limited) from postcompression of 25 fs (Fourier limit) amplified pulses in an argon-filled HCF. The d-scan technique was used to measure the reference pulse required by STARFISH and the latter was applied for the spatiotemporal characterization of the mode compressed at the output of the fiber, where the pulse structure and spatial chirp were studied. These pulses were also focused using an off-axis parabolic mirror and measured around the focal spot in the spatiotemporal domain. The characterization of intense ultra-broadband post-compressed pulses presents additional difficulties due to the higher energy, larger spectral bandwidth, stronger phase modulations, and higher instability compared to pulses delivered by an oscillator.

The full spatiotemporal characterization of intense fewcycle lasers provides useful information for the study of the dynamics and characteristics of filamentation [17, 30] and HCF post-compressed pulses. In future experiments, it may be used to tackle the comparison between both postcompression techniques. This information is relevant for the optimization of the process itself and for applications (e.g., HHG) of the generated pulses.

\section{Experimental setup for post-compression and spatiotemporal characterization}

We post-compressed pulses delivered by a $1-\mathrm{kHz} \mathrm{Ti}$ :sapphire CPA amplifier (Femtolasers FemtoPower Compact PRO CEP) in a gas-filled HCF and chirped mirror compressor. The complete experimental setup is depicted in Fig. 1. The amplified pulses, with a Fourier-transform limit (FTL) of $25 \mathrm{fs}$, were coupled in the hollow fiber with a 1.5-m focal length lens. The HCF had an inner diameter of $250 \mu \mathrm{m}$, a length of $1 \mathrm{~m}$, and was filled with argon at a pressure of 960 mbar. The pulse energy before the HCF was $375 \mu \mathrm{J}$ and at the output of the fiber was $150 \mu \mathrm{J}$ (transmission of $40 \%$ ). The chirp of the input pulse on the $\mathrm{HCF}$ was adjusted in the amplifier compressor to optimize the spectral broadening (Fig. 3c) and the transverse mode profile (Fig. 2b) at the fiber output. Additionally, we also optimized the fiber output with an iris ( $7 \mathrm{~mm}$ diameter) placed just before the lens for the fine control of the input energy and the input mode being coupled in the HCF (Fig. 2a). Moreover, the post-compression was optimized for a very stable output mode, as required both for subsequent applications and for (multi-shot) pulse characterization. The quality of the d-scan and STARFISH traces is an indication of this stability, since shot-to-shot variations would strongly affect them. In parallel, the raw d-scan traces already provided a fast diagnosis to ensure that the temporal profile corresponded to clean compressed pulses without satellites that could occur in the $\mathrm{HCF}$, given that it only takes a few seconds to acquire them and the shape of the trace gives direct, interpretable information about the structure of the pulse [26]. Owing to the nonlinearity of the argon gas inside the HCF, the input spectrum was broadened to a 4.1-fs FTL spectrum extending from 540 to $990 \mathrm{~nm}$ (Fig. 3c). A spherical silver mirror at near-normal incidence $(\mathrm{ROC}=3,000 \mathrm{~mm}$ ) was placed after the HCF to collimate the spectrally broadened pulses. To complete the post-compression of the pulses, a compressor made of a glass wedge pair and a set of ultra-broadband chirped mirrors (CMs) was used. The wedges (Femtolasers GmbH) were made of BK7 with an antireflection coating and an angle of $8^{\circ}$. The CMs (UltraFast Innovations $\mathrm{GmbH}$ ) are designed in such a way that when two bounces are combined, with incidence angles of $5^{\circ}$ and $19^{\circ}$, respectively, the residual group delay oscillations are minimized [31]. 


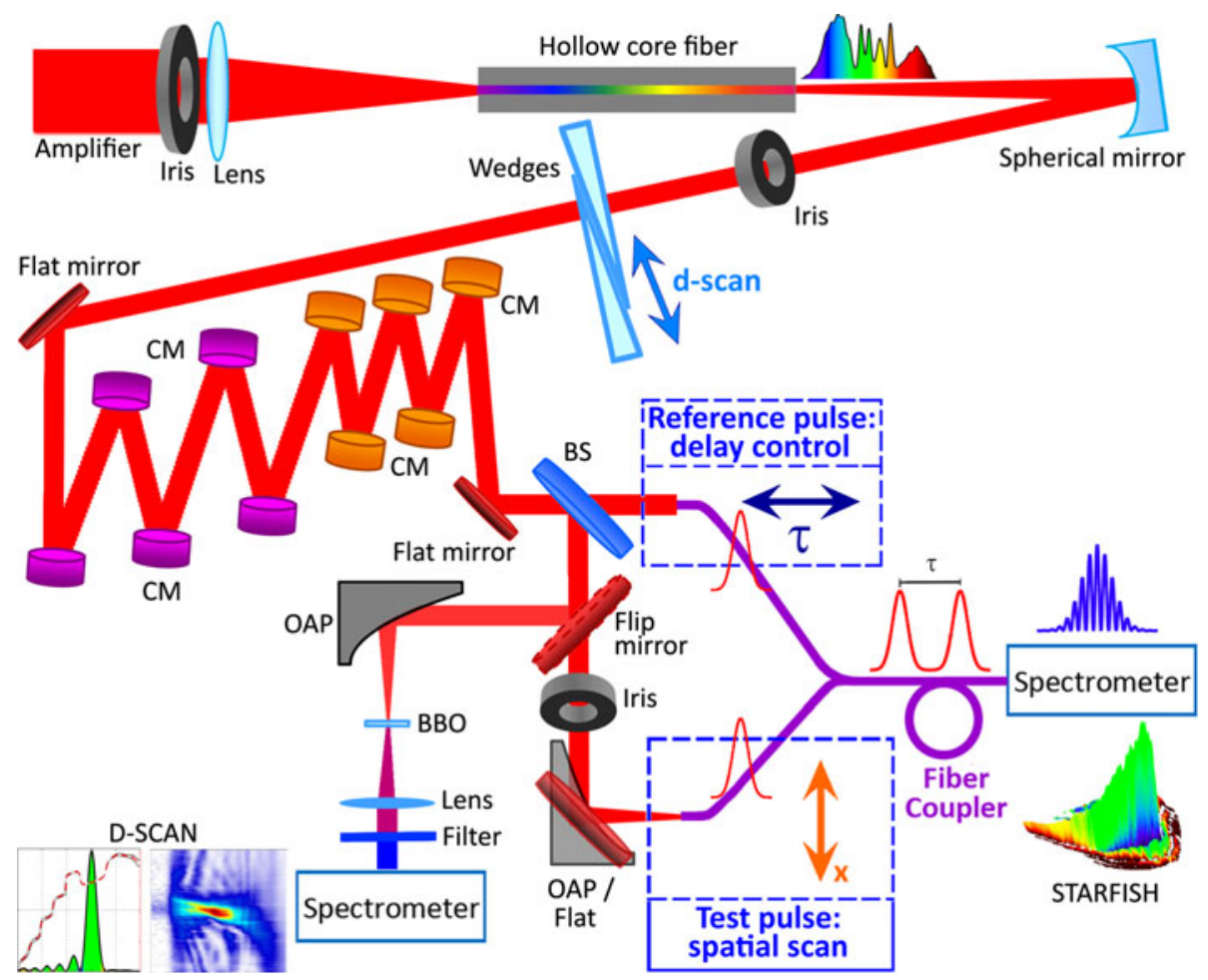

Fig. 1 Experimental setup for the generation and spatiotemporal characterization of post-compressed pulses. The amplified pulses are spectrally broadened in a hollow-fiber and the output mode is collimated with a spherical mirror. The pulses are divided with a dispersion-balanced broadband 50/50 beam splitter $(B S)$ to perform the spectral interferometry of STARFISH. Using a flip mirror, the reflected pulses can be simultaneously compressed and characterized by dispersion scan $(d$-scan $)$ where a compressor made of a pair of glass wedges and 5 pairs of chirped mirrors $(C M)$ is used to track the SHG signal generated in a nonlinear crystal $(B B O)$ as a function of wedge insertion. The test and reference pulses are then combined in a fiber optic coupler and sent to the spectrometer. The position of the test fiber is scanned over the spatial coordinate ( $x$-axis). The hollowfiber output (test pulse) is measured unfocused (flat mirror) and focused (off-axis parabola, OAP, $5 \mathrm{~cm}$ focal length)
Fig. 2 a Input mode coupled at the entrance of the $\mathrm{HCF}$ with an inner diameter of $250 \mu \mathrm{m}$.

b Spatial profile of the output mode of the HCF after collimation with the spherical mirror
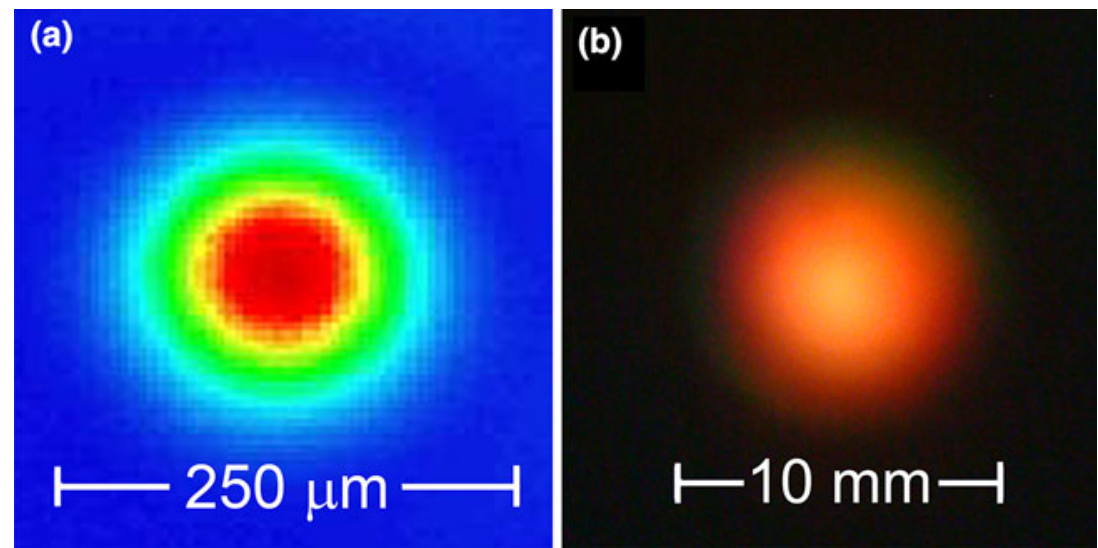

We used five pairs of mirrors for a total number of five bounces at each angle, as illustrated in Fig. 1 (we have identified the CMs with different colors, orange and purple, for $5^{\circ}$ and $19^{\circ}$ incidence, respectively). The nominal group delay dispersion (GDD) of the CMs was around $-50 \mathrm{fs}^{2}$ per bounce at $800 \mathrm{~nm}$. The variable insertion of one of the wedges allowed us to fine-tune the ultimate post- compressed duration of the pulses, and was also used as part of the d-scan technique as described below.

For the characterization of the post-compressed pulses, we used the STARFISH technique [28] assisted by the d-scan technique [26] to measure the reference pulse, in a configuration recently introduced for few-cycle pulses [29]. A replica of the pulse to be characterized was created with a dispersion- 
Fig. 3 a Experimental and b retrieved d-scan traces of the reference pulse. c Spectral intensity (black) and phase (dashed red) of the retrieved pulse. d Temporal intensity (black) and phase (dashed red) of the reference pulse. The gray curves in $\mathbf{c}$ and $\mathbf{d}$ represent the standard deviation of the spectral phase, and of the temporal intensity and phase, respectively. The intensity profile (d) is color-filled by the instantaneous wavelength following the same color scale than in $\mathbf{c}$
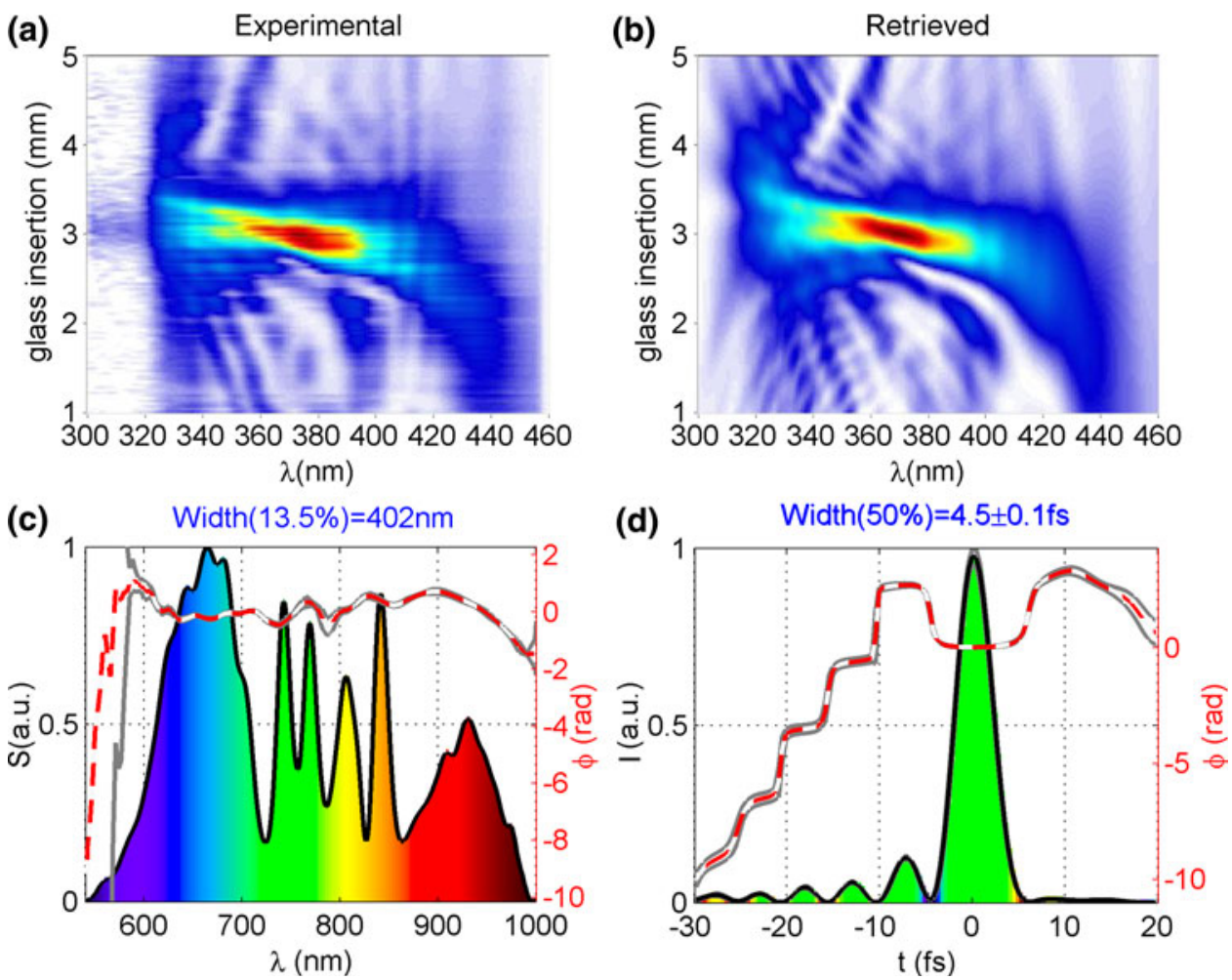

balanced (same dispersion in the reflected and transmitted beams) broadband beam splitter $(600-1,500 \mathrm{~nm})$, BS (Venteon $\mathrm{GmbH}$ ). One pulse was measured with d-scan (after the flip mirror) and subsequently used as the reference pulse in STARFISH.

The experimental implementation of d-scan consists in measuring the second harmonic generation (SHG) spectrum of the pulse while the dispersion is varied via the translation of one of the wedges around the point of maximum compression (minimum pulse duration). As a result, a spectrally resolved SHG trace as a function of wedge insertion (dispersion) is obtained. In the present setup, we focus the pulses with an off-axis parabolic (OAP) mirror (focal length of $5 \mathrm{~cm}$ ) in a nonlinear crystal (BBO, $20 \mu \mathrm{m}$ thick, cut for type I SHG at $800 \mathrm{~nm}$ ). The SHG signal is collimated with a lens and a blue filter is used to remove the remaining fundamental frequency signal before detection with the spectrometer (HR4000, Ocean Optics Inc.).

In STARFISH, a single-mode, $4 \mu \mathrm{m}$ core diameter, broadband fiber optic coupler $[28,29]$ is used to combine the reference pulse (already characterized by d-scan) and the (unknown) test pulse. A spectral interferogram (SI) of the pulses with a relative delay $\tau$ is measured in a standard fiber-coupled spectrometer (S2000, Ocean Optics Inc.). This information will allow us to obtain the temporal reconstruction of the pulses. The test pulse fiber input is scanned over the spatial profile of the pulse in one axis
( $x$-scan), where cylindrical symmetry is assumed. This allows for the spatiotemporal characterization of the pulses.

The spatiotemporal STARFISH characterization of the few-cycle pulses is first performed directly after the CM setup, so a flat mirror is used to direct the pulses to the test fiber. This mirror was then replaced with a $5-\mathrm{cm}$ focal length OAP to study the focusability of the pulses, and to characterize them around the focal region.

Before the wedges and CM compressor, an iris of $10-\mathrm{mm}$ diameter is used to select the spatial mode after collimation of the fiber output (see Fig. 2b). On account of the 10-mm iris before the wedges and the losses inside the compressor itself, the pulse energy decreased from 150 to $90 \mu \mathrm{J}$ before the BS. The beam was not perfectly collimated after the spherical mirror, since it diverged more than would have been expected for a collimated beam with the same waist. In fact, the beam size of the pulses increased up to $13 \mathrm{~mm}$ just before the last mirror prior to the test fiber (the optical path in the $\mathrm{CM}$ setup was $169 \mathrm{~cm}$ ). At this position, we selected the spatial mode with a diaphragm to eliminate the residual (mostly conical) emission around the main portion of the beam.

The spectral phase of the reference pulse is retrieved by the d-scan technique by optimizing the simulated SHG trace compared with the experimental trace using an iterative algorithm [26]. The spatiotemporal amplitude and phase characterization is obtained by an algorithm based on 
the Fourier-transform of the spectral interferences given by the STARFISH technique [28].

\section{Spatiotemporal analysis of sub-5-fs pulses after hollow-fiber post-compression}

\subsection{Characterization of the reference pulse using d-scan}

As said before, the spectrally broadened amplified pulses are compressed with five pairs of broadband chirped mirrors and a pair of BK7 glass wedges. This compressor is also a part of the d-scan technique setup for the characterization of the reference pulse, which is required for the spectral interferometry. The d-scan trace was taken by measuring the SHG signal while varying the glass insertion. The total range of insertion (in the propagation direction) was $d=4.34 \mathrm{~mm}$ using a lateral insertion step of $0.215 \mathrm{~mm}$, which corresponds to 146 sampling points. The resulting experimental d-scan trace is shown in Fig. 3a.

The d-scan algorithm was executed five times with different input conditions (five different starting guesses) in order to ensure the convergence of the retrieved phase. The retrieved trace is given in Fig. $3 b$, and is in very good agreement with the experimental trace. The experimental spectrum and the retrieved phase of the pulse for maximum compression are shown in Fig. 3c. The full width at $1 / e^{2}$ of the maximum (in intensity) of the hollow-fiber spectrum is $402 \mathrm{~nm}$. The standard deviation (SD) of the phase (gray curves) for the different runs of the algorithm provides information on the precision error. The spectral phase retrieved is precise except for the shorter wavelengths owing to the experimental d-scan trace being cropped in the bluer part of the spectrum, as will be explained below. In Fig. 3d, the retrieved temporal intensity and phase of the corresponding pulse are represented. The optimum spectral phase has a small contribution of negative third-order dispersion that produces the pre-pulses. The corresponding SDs (gray curves) are very small, which means that the spectral phase deviation for shorter wavelengths hardly affects the temporal retrieval. The duration of the pulse is $4.5 \pm 0.1$ fs (intensity FWHM), close to its FTL of 4.1 fs. The carrier wavelength calculated from the center of gravity of the spectral power density (in frequency) is $\lambda_{\mathrm{g}}=739 \mathrm{~nm}$. The temporal intensity in Fig. 3d has been color-filled with the instantaneous wavelength of the pulse $\lambda_{t}$. From the tilt of the phase in the pre-pulses, we see that they are slightly redder than the main pulse (centered at $\lambda_{t}=739 \mathrm{~nm}$ ), as illustrated by the color fill. The small deviation of the instantaneous wavelength from the carrier wavelength is an evidence of the good compression achieved. The main reason why the final pulse duration deviates from the FTL is the divergence in the spectral phase introduced by the broadband beamsplitter for wavelengths below $600 \mathrm{~nm}$, since this element is designed to work above this wavelength.

The lack of signal below $320 \mathrm{~nm}$ in the experimental trace may be partly due to the cut-off of the blue filter and by UV absorption in the collimating lens. This justifies the difference between measured and retrieved traces in that spectral region. To minimize this effect, a spatial mask can be used instead of the filter or lens [27]. The phase distortion introduced by the beamsplitter for wavelengths below $600 \mathrm{~nm}$ may also contribute to the smaller SHG signal observed at shorter wavelengths. In spite of this, thanks to the trace redundancy in the d-scan technique, it is possible to recover phase information for regions where no SHG signal has been measured at all, as was shown in [26, 29].

As mentioned above, the optimal pulse compression with this system is close to the FTL. To further analyze this compression, we calculated the chronocyclic or time-frequency Wigner distribution function [32] of the pulse and the FTL of the spectrum. For a certain function defined in the time domain (in our case the electric field of the pulses, $E(t)$ ), the Wigner distribution can be interpreted as the probability (despite it taking positive and negative values) to find a certain wavelength (or frequency $\omega$ ) at a given time $t$, i.e., it gives us the spectral distribution within the pulse. The definition of the time-frequency Wigner distribution function $W_{\mathrm{T}}$ is $[32,33]$

$W_{\mathrm{T}}(t, \omega)=\int_{-\infty}^{\infty} E\left(t+\frac{t^{\prime}}{2}\right) E *\left(t-\frac{t^{\prime}}{2}\right) \mathrm{e}^{-i \omega t^{\prime}} \mathrm{d} t^{\prime}$.

The calculations for the pulse retrieved by the d-scan and for the FTL of the spectrum are shown in Fig. 4a, b, respectively. The information given by $W_{\mathrm{T}}$ is related to the instantaneous frequency (see Fig. 3d), although $W_{\mathrm{T}}$ provides the whole information of the temporal distribution of wavelengths, in contrast to the single value of the effective instantaneous wavelength. This gives further insight into the pulse structure and compression, and can give a visual and intuitive idea of how far we are from the FTL by comparing the Wigner distributions of the retrieved pulse and of its FTL (Fig. 4a, b). In our case, since most of the spectrum is contained in the main peak of the pulse (like in the FTL pulse, except for the tails of the spectrum), this means that almost the whole spectrum is well compressed (i.e., its spectral phase is well compensated for). Moreover, when comparing the scales of the plots, a small loss of signal occurs in the measured pulse with respect to its FTL. This is in 
Fig. 4 Wigner distribution functions and corresponding marginals for the electric field of the a experimental pulse and b Fourier-transform limit of the spectrum. The two functions are represented in the same (arbitrary) units in order to compare the respective signal strength
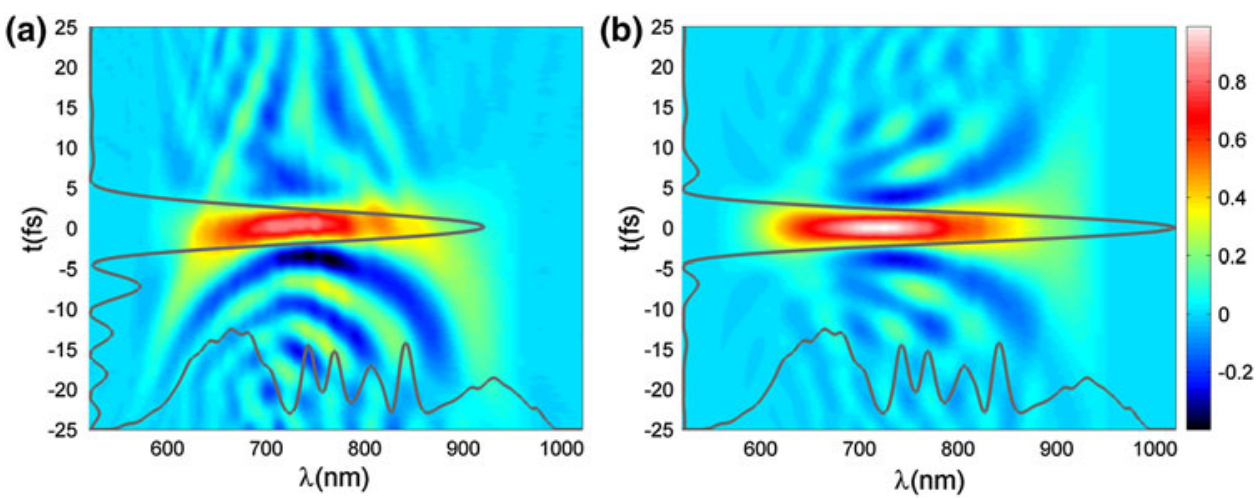

agreement with the fact that the peak intensity of the pulse for the d-scan retrieval is 0.8 times the FTL peak intensity. The marginals of $W_{\mathrm{T}}$ are also given in the plots, where integration of the Wigner function of the pulse over the frequency and time axes provides the temporal intensity (left) and power spectral density (bottom), respectively.

\subsection{Spatiospectral and spatiotemporal characterization of the output mode using STARFISH}

The output mode of the post-compression of the pulses in the $\mathrm{HCF}$ was characterized in the spatiotemporal domain. The spatial profile was scanned with the fiber across the $13-\mathrm{mm}$ diameter of the pulse with steps of $50 \mu \mathrm{m}$ (261 sampling points). The spatially resolved spectrum (Fig. 5a) shows that the spectral distribution is fairly constant across the $x$ - coordinate, only presenting less broadening in the bluer part of the spectrum for the outermost part of the spatial profile. The frequency-resolved wavefront (Fig. 5b) presents a curvature responsible for the beam divergence (already observed during beam propagation), which will be taken into account to simulate the focus of the pulse in Sect. 3.3.

The pulse-front curvature in the spatiotemporal intensity (Fig. 5d) corresponds to the expected curvature of a diverging beam. It exhibits a relatively small variation of $\approx 30 \mathrm{fs}$ from the center to the periphery of the beam ( $\approx 13 \mathrm{~mm}$ diameter), although it is large compared to the pulse duration. Nevertheless, we will see that the presence of wavefront and pulse-front curvature do not compromise the focusability of the beam and a tight focus is achieved. The spatiotemporal intensity is shown in Fig. 5e, colorfilled with the instantaneous wavelength to better illustrate
Fig. 5 Pulse post-compressed in an $\mathrm{HCF}$ experiment: a normalized spatiospectral intensity and $\mathbf{b}$ frequencyresolved wavefront, the latter represented in different colored curves for each wavelength (see the color bar). c Fourier limit (blue) and retrieved pulse duration (red). d Normalized spatiotemporal intensity and e same as $\mathbf{d}$, but color-filled with the instantaneous wavelength (see the color bar). f Instantaneous wavelength at the pulse-front (blue) and center wavelength (red)
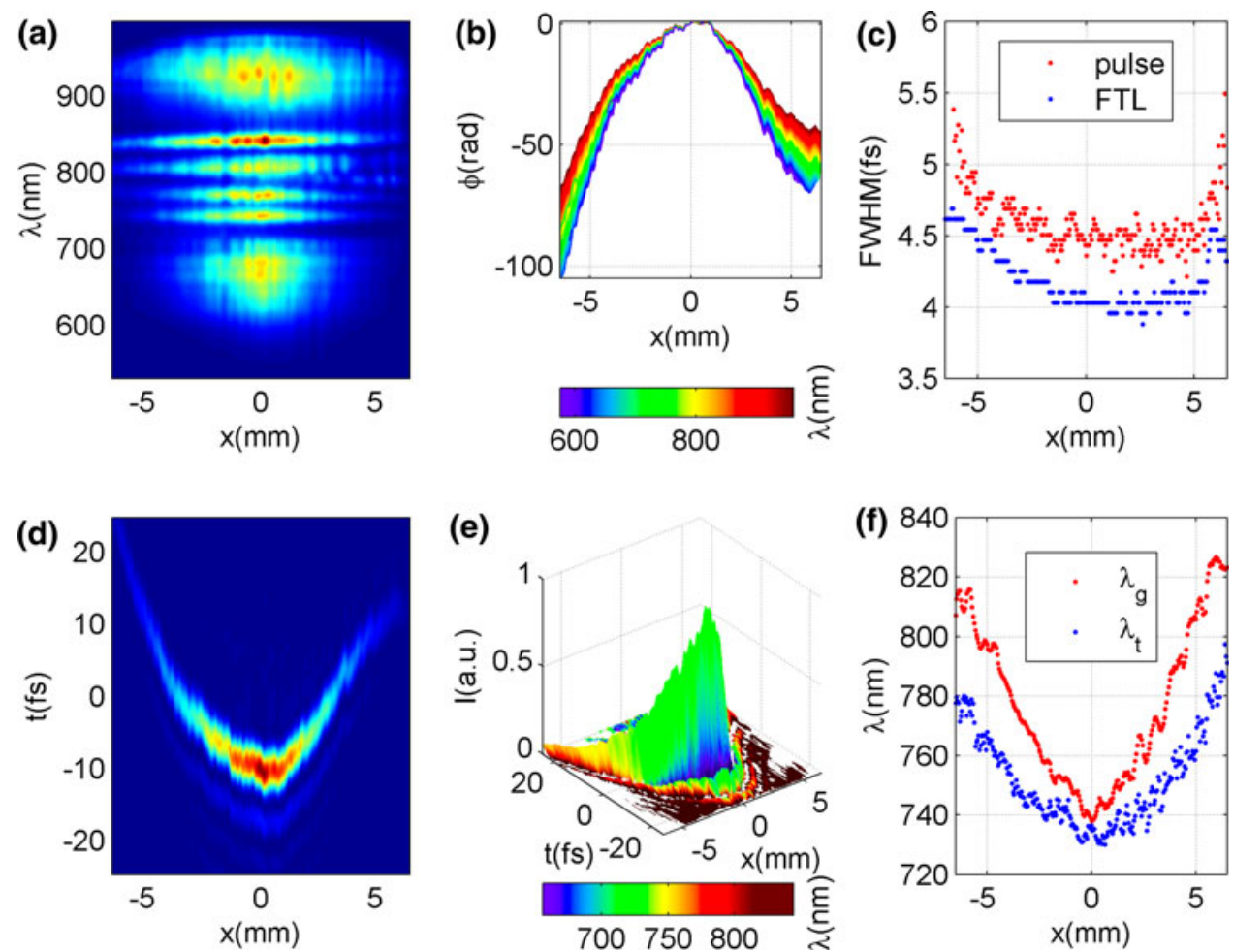

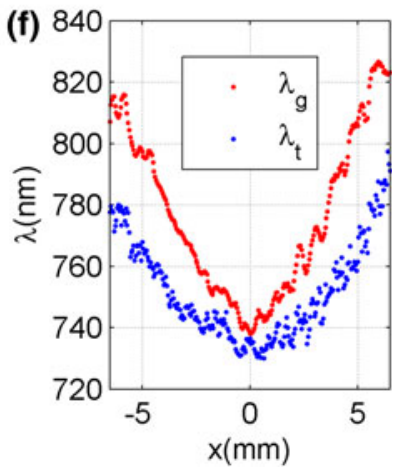


the spatial dependence of the temporal chirp. The main peak color shifts from bluer to redder values as we move away from the center of the pulse $(x=0)$, as expected from the spatially resolved spectrum in Fig. 5a.

The pulse duration (intensity FWHM) as a function of $x$ is presented in Fig. 5c for the retrieved pulse and for the FTL of the spectrum. The pulse duration varies approximately from 4.5 fs on-axis to 5.0 fs in the wings, whereas the FTL varies from 4.0 to 4.5 fs. In Fig. 5 f, we show the carrier wavelength dependence on $x$, both for the center of gravity of the spectrum $\left(\lambda_{\mathrm{g}}\right)$ and the instantaneous wavelength $\left(\lambda_{t}\right)$ evaluated at the maximum of the pulse (i.e., at the pulse-front). We see that $\lambda_{\mathrm{g}}$ varies from $740 \mathrm{~nm}$ (onaxis) to $820 \mathrm{~nm}$ (wings), whereas $\lambda_{t}$ varies from $735 \mathrm{~nm}$ (on-axis) to $790 \mathrm{~nm}$ (wings). These results are in agreement with previous works, where blue-shift and larger spectral broadening and pulse shortening were observed on-axis in comparison with the outer part of the spatial profile [19]. Redder pre-pulses with the same curvature than the main pulse (the pulse-front) are observed, similarly to the reference pulse. There is almost no chromatic aberration in the wavefront (Fig. 5b) since all wavelengths have practically the same curvature, except for the intrinsic wavenumber dependence $\phi\left(x_{0}, \lambda\right) \propto k \propto \lambda^{-1}$ (similar to the results given in [34] for a focusing refractive lens), as we will calculate in the next section.
3.3 Spatiospectral and spatiotemporal characterization of the focus using STARFISH

The pulses were focused with an $\mathrm{OAP}(f=5 \mathrm{~cm})$. The focus was spatially resolved with the test fiber, which scanned the transverse profile across $30 \mu \mathrm{m}$ in steps of $1 \mu \mathrm{m}$ ( $x$-axis). Despite the $4 \mu \mathrm{m}$ fiber core diameter, we have demonstrated in a previous work that using a smaller step allows us to recover the structure and size of focused pulses [34]. In this experiment, a neutral-density filter was placed before the BS (note that the dispersion before the interferometer is compensated in the SI) to avoid damage or nonlinear effects in the collecting fiber, so the linear focus could be characterized. The spectrum as a function of the $x$-coordinate (Fig. 6a) presents a spatial chirp, with increasing red-shift for increasing values of $x$. The spatiotemporal distribution corresponds to a well-defined focus in space and time (Fig. 6d), with a spatial width of $10 \mu \mathrm{m}$ (FWHM) and a temporal duration on-axis of $4.5 \mathrm{fs}$ (FWHM). The instantaneous wavelength combined with the intensity (Fig. 6e) inherits the spatial chirp from the frequency domain (shown in Fig. 6a). Since the input pulse is symmetric in $x$ (Fig. 5), the spatial chirp may be originated by a slight misalignment in the OAP. The frequencyresolved wavefronts (Fig. 6b) show a slight divergence, meaning that the measurement was not taken exactly at the focus, but just before it. Also, the wavefronts for the different wavelengths have a gradual relative tilt, which is an
Fig. 6 Post-compressed pulses focused with an OAP; experiment: a normalized spatiospectral intensity and b frequency-resolved wavefront, the latter represented in different colored curves for each wavelength (see the color bar). c Fourier limit (blue) and pulse duration ( $r e d)$.

d Normalized spatiotemporal intensity and e same as $\mathbf{d}$, colorfilled by the instantaneous wavelength (see the color bar). f Instantaneous wavelength at the pulse-front (blue) and center wavelength (red)
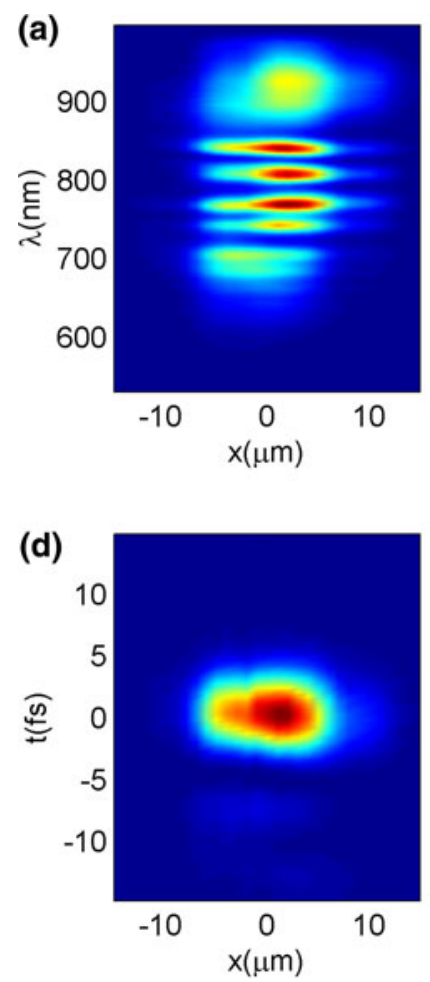
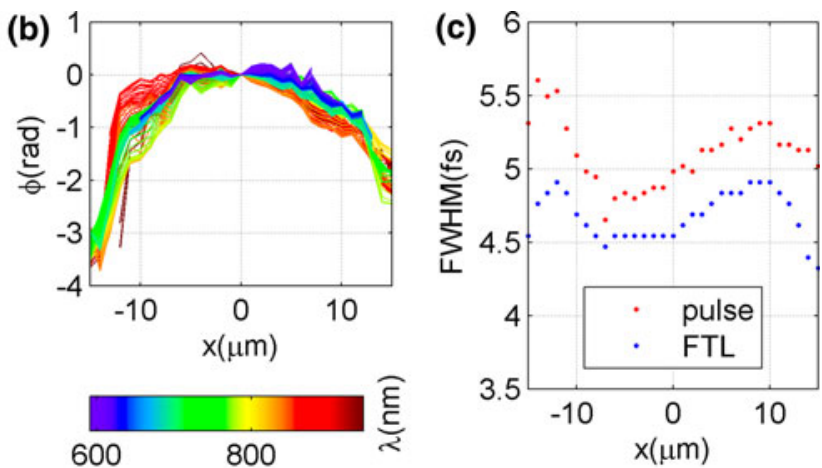

(e)

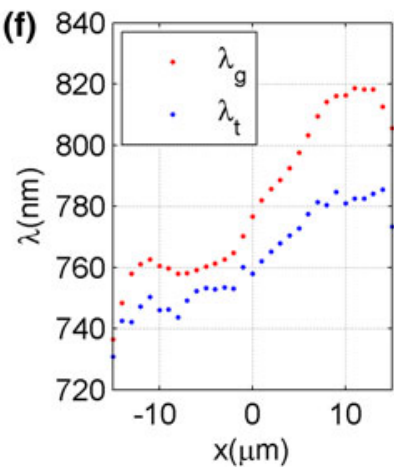


evidence of the spatial chirp originated by the asymmetric focusing phase introduced by the misalignment.

The FWHM intensity of the Fourier-transform limited (FTL) pulse increases along the $x$-axis from 4.5 to 4.9 fs and the retrieved pulse duration from 4.8 to $5.3 \mathrm{fs}$ (Fig. 6c). Similar behavior is observed for the carrier (central) wavelength $\lambda_{\mathrm{g}}$ that varies from 760 to $810 \mathrm{~nm}$ (Fig. 6f). Again, the instantaneous wavelength at the pulsefront, $\lambda_{t}$, is blue-shifted with respect to $\lambda_{\mathrm{g}}$.

We studied the effect of the spatiospectral phase (or wavefront) of the mode before focusing (Fig. 5b). This phase is mainly quadratic and can be written as $\phi(x ; \lambda)=$ $(\pi / \lambda)\left(x^{2} / f_{\text {in }}\right)$, corresponding to a diverging beam with a focal length $f_{\text {in }}$. From the fit to the phase $\phi(x ; \lambda)$ for each wavelength, we extracted the focal length $f_{\text {in }}=$ $-4,724 \pm 26 \mathrm{~mm}$ (regression coefficient $R=0.9989$ ). When combined with the focal length of the OAP, $f_{\mathrm{OAP}}=50 \mathrm{~mm}$, we obtained the effective focal length $f_{\text {eff }}=50.53 \mathrm{~mm}$. Assuming that only this quadratic phase is present, this will simply cause a shift in the focal position along the propagation axis, but higher order curvature terms in the wavefront may distort the focal spot.

Finally, we analyzed the effect of the numerical aperture (NA) of the fiber coupler (the test pulse fiber arm). In a previous work we measured a coupling efficiency of $50 \%$ at an angle of incidence of $\theta=5^{\circ}$ [29]. Here, we used the experimental dependence of the coupling efficiency on the angle $\theta$, denoted by $T(\theta)$, to study its effect in the measurement of the focus of the OAP. In the ray-tracing approximation, the angle $\theta$ is translated to the input spatial plane as $\tan \theta \approx r / z$, where $z=f$ for observation at the focus (this gives the function $T(r)$ plotted in Fig. 7a). In the present case, the spatial intensity profile has a Gaussianlike shape, so the less efficiently coupled part of the profile, the periphery, is the part with the least contribution. Figure $7 \mathrm{a}$ shows the spatial intensity profile $I(r)$ for the almost collimated beam, the fiber transmission $T(r)$, and the modified spatial profile $I(r) \cdot T(r)$ considering raytracing. The experimental spatially resolved spectrum before the OAP (collimated beam) is shown in Fig. 7b (extracted from Fig. 5a), which can be compared with the same magnitude modified by $T(r)$ in Fig. 7c (this plot illustrates the spectrum of the focused pulse that would be collected due to the numerical aperture of the fiber). From Fig. 7 it can be seen that the most affected part is the periphery of the spatial profile.

The distribution of the orientation of the wave vectors inside a monochromatic Gaussian beam using the spatial Wigner distribution function is discussed in depth in [35]. Far from the Rayleigh zone, it corresponds to a well-defined angle, which can be simply calculated by ray-tracing, as a function of the transverse spatial coordinate. Since larger angles occur in the periphery of the beam, outside the focal region, the angular filtering of the NA of the fiber results in a reduction of the spatial width, which can be estimated by the correction explained in the previous paragraph (see Fig. 7). Conversely, at the focus position all the wave vectors (from the ray-tracing) overlap and the wave vector spreading is independent from the spatial coordinate, so the ray-tracing approximation is obviously unacceptable there [35]. For this reason, in the focus (where we measured the pulse), the effect of the NA coupling will be ideally a reduction in the collected signal without spatial distortion. In our case, the NA of the pulse is slightly lower than the NA of the detection fiber (see the curves $I(r)$ and $T(r)$ in Fig. 7, respectively), so the effect of the NA of the fiber on the spatiotemporal measurements will be small (Fig. 7). The modification (decrease in spatial width) in the spatiospectral domain is illustrated by comparing Fig. 7b (actual) and Fig. 7c (modified), which corresponds in the spatial domain (after wavelength integration) to the comparison between the curves $I(r)$ and $I(r) \cdot T(r)$, respectively. Note that this estimation of the profile modification corresponds to the worst-case scenario, in which ray-tracing can be (a)

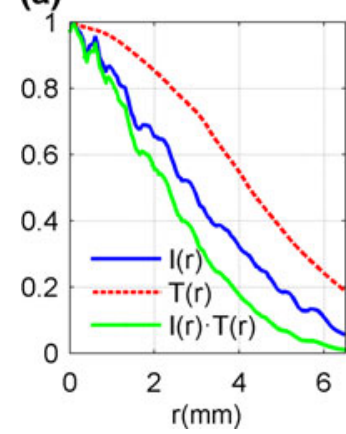

(b)

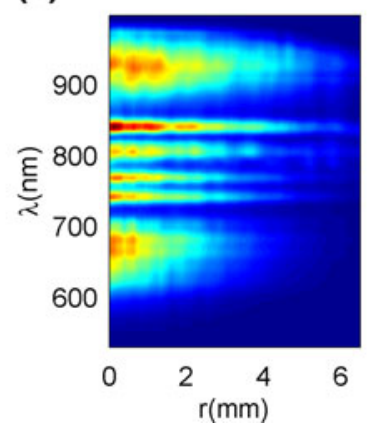

(c)

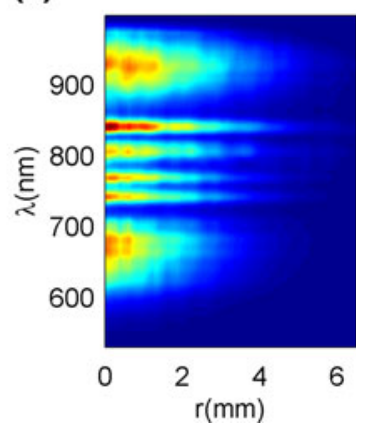

Fig. 7 a Spatial profile of the collimated pulse (blue), transmission of the fiber due to the numerical aperture (red) and corrected spatial distribution (green). b Experimental spatially resolved spectrum of the collimated pulse. c Calculated spatially resolved spectrum [b modified by $T(r)$ ], corresponding to the spectrum of the focused pulse that would collect the fiber owing to the numerical aperture 
applied (out of the Rayleigh zone), so the experimental measurement of the focus (Fig. 6) is very close to reality.

The present analysis will be more complex in the case of polychromatic non-Gaussian beams, which may also be inhomogeneous and present wavefront aberrations. This will cause a less predictable propagation (if the unfocused pulse is known, numerical simulations can still be performed). However, ray-tracing can still give a first approximation of the wave vector distribution and an upper bound for the maximum angle collected $\theta_{\max }$ can be estimated by the relation $\tan \theta_{\max } \simeq r_{\max } / f$, where $r_{\max }$ is the radius of the unfocused pulse and $f$ is the focal length. Naturally, the effect of the NA will not be felt by smaller beams or longer focal lengths. Finally, the rather homogeneous mode at the output of the hollow-fiber plays to our advantage in the sense that distortions (due to the NA) at the focus of the beam will be reduced.

\section{Conclusions}

We have produced sub-two-cycle pulses by post-compression of 25-fs pulses in a hollow-core fiber. The postcompression was optimized to have an ultra-broadband spectrum corresponding to few-cycle pulses (4.1 fs Fourier limit) and a homogeneous (near-Gaussian) spatial profile with a significantly stable mode. The pulse energy at the output of the hollow-fiber was $150 \mu \mathrm{J}$. Optimum compression was achieved by compensating the spectral phase with a pair of wedges and ultra-broadband chirped mirrors.

The post-compressed pulses were characterized in the spatiotemporal domain using STARFISH technique, and the reference pulse was measured with the d-scan technique. We first studied the output mode of the hollow-fiber and found that the spectral broadening and the blue-shift are significantly larger at the center $(x=0)$ of the pulse than in the periphery. This resulted in an increase in pulse duration from $4.5 \mathrm{fs}$ at the beam center up to $5 \mathrm{fs}$ at the periphery. A symmetric spatial chirp (relative to $x=0$ ) was consequently observed in the spatiotemporal reconstruction. Then, we characterized the pulse focused with an off-axis parabolic mirror $(f=5 \mathrm{~cm})$, which produced a measured focal spot size of $10 \mu \mathrm{m}$ (FWHM).

The conjunction of the techniques d-scan and STARFISH for the measurement of ultra-broadband, intense 4.5 fs pulses (4.1 fs FTL) provides an efficient means for completely characterizing pulses in the range of the lower pulse duration measurable by state-of-the-art temporal characterization techniques in the near-infrared region. The large bandwidth of the critical elements involved in the detection of STARFISH-i.e., beam splitter and fiber coupler-and the relaxed bandwidth requirements of d-scan are vital to reach this regime. The possibility of measuring high-energy, low repetition rate pulses in this range is promising for applications, such as strong-field experiments or the study and optimization of filament and hollow-fiber compressed pulses.

Acknowledgments We acknowledge support from Spanish Ministerio de Ciencia e Innovación (MICINN) through the Consolider Program SAUUL (CSD2007-00013), Research projects FIS200909522, and grant programs Formación de Profesorado Universitario (No. AP2007-00236 for B. Alonso) and Ramón y Cajal (for I.J. Sola); and from the Junta de Castilla y León through the Program for Groups of Excellence (GR27). H. Crespo acknowledges Fundos FEDER, through Programa Operacional Factores de Competitividade-COMPETE, and Fundação para a Ciência e a Tecnologia (FCT) under grants No. PTDC/FIS/115102/2009 and PTDC/FIS/122511/2010. M. Miranda and F. Silva acknowledge FCT and FEDER grants No. SFRH/BD/37100/2007 and SFRH/BD/69913/2010, respectively. M. Miranda also acknowledges support from the European Research Council (grant ALMA) and the Swedish Research Council. We also acknowledge support from Centro de Láseres Pulsados (CLPU), Salamanca, Spain.

\section{References}

1. A. Baltuška, Th Udem, M. Uiberacker, M. Hentschel, E. Goulielmakis, Ch. Gohle, R. Holzwarth, V.S. Yakovlev, A. Scrinzi, T.W. Hänsch, F. Krausz, Nature 421, 611-615 (2003)

2. S. Baker, J.S. Robinson, C.A. Haworth, H. Teng, R.A. Smith, C.C. Chirilã, M. Lein, J.W. Tisch, J.P. Marangos, Science 312, 424-427 (2006)

3. K.W.D. Ledingham, P. McKenna, R.P. Singhal, Science 300, 1107-1111 (2003)

4. T. Brabec, F. Krausz, Rev. Mod. Phys. 72, 545-591 (2000)

5. M. Hentschel, R. Kienberger, Ch. Spielmann, G.A. Reider, N. Milosevic, T. Brabec, P. Corkum, U. Heinzmann, M. Drescher, F. Krausz, Nature 414, 509-513 (2001)

6. Y. Mairesse, A. de Bohan, L.J. Frasinski, H. Merdji, L.C. Dinu, P. Monchicourt, P. Breger, M. Kovačev, R. Taïeb, B. Carré, H.G. Muller, P. Agostini, P. Salières, Science 302, 1540-1543 (2003)

7. G. Sansone, E. Benedetti, F. Calegari, C. Vozzi, L. Avaldi, R. Flammini, L. Poletto, P. Villoresi, C. Altucci, R. Velotta, S. Stagira, S.D. Silvestri, M. Nisoli, Science 314, $443-446$ (2006)

8. I.J. Sola, E. Mevel, L. Elouga, E. Constant, V. Strelkov, L. Poletto, P. Villoresi, E. Benedetti, J.P. Caumes, S. Stagira, C. Vozzi, G. Sansone, M. Nisoli, Nat. Phys. 2, 319-322 (2006)

9. A.A. Eilanlou, Y. Nabekawa, K.L. Ishikawa, H. Takahashi, K. Midorikawa, Opt. Express 16, 13431-13438 (2008)

10. S. Witte, R. Zinkstok, W. Hogervorst, K. Eikema, Opt. Express 13, 4903-4908 (2005)

11. M. Nisoli, S. De Silvestri, O. Svelto, R. Szipöcs, K. Ferencz, Ch. Spielmann, S. Sartania, F. Krausz, Opt. Lett. 22, 522-524 (1997)

12. B. Schenkel, J. Biegert, U. Keller, C. Vozzi, M. Nisoli, G. Sansone, S. Stagira, S. De Silvestri, O. Svelto, Opt. Lett. 28, 1987-1989 (2003)

13. C.P. Hauri, W. Kornelis, F.W. Helbing, A. Heinrich, A. Mysyrowicz, J. Biegert, U. Keller, Appl. Phys. B 79, 673-677 (2004)

14. A. Zaïr, A. Guandalini, F. Schapper, M. Holler, J. Biegert, L. Gallmann, A. Couairon, M. Franco, A. Mysyrowicz, U. Keller, Opt. Express 15, 5394-5404 (2007)

15. D. Faccio, A. Lotti, A. Matijosius, F. Bragheri, V. Degiorgio, A. Couairon, P. Di Trapani, Opt. Express 17, 8193-8200 (2009)

16. J. Odhner, R.J. Levis, Opt. Lett. 37, 1775-1777 (2012)

17. B. Alonso, I.J. Sola, J. San Román, O. Varela, L. Roso, J. Opt. Soc. Am. B 28, 1807-1816 (2011) 
18. X. Sun, S. Xu, J. Zhao, W. Liu, Y. Cheng, Z. Xu, S.L. Chin, G. Mu, Opt. Express 20, 4790-4795 (2012)

19. L. Gallmann, T. Pfeifer, P.M. Nagel, M.J. Abel, D.M. Neumark, S.R. Leone, Appl. Phys. B 86, 561-566 (2007)

20. T. Witting, F. Frank, W.A. Okell, C.A. Arrell, J.P. Marangos, J.W.G. Tisch, J. Phys. B 45, 74014 (2012)

21. I.A. Walmsley, C. Dorrer, Adv. Opt. Photon. 1, 308-437 (2009)

22. A. Baltuška, M.S. Pshenichnikov, D.A. Wiersma, Opt. Lett. 23, 1474-1476 (1998)

23. A.S. Wyatt, I.A. Walmsley, G. Stibenz, G. Steinmeyer, Opt. Lett. 31, 1914-1916 (2006)

24. J.R. Birge, H.M. Crespo, F.X. Kärtner, J. Opt. Soc. Am. B 27, 1165-1173 (2010)

25. J. Itatani, F. Quéré, G.L. Yudin, MYu. Ivanov, F. Krausz, P.B. Corkum, Phys. Rev. Lett. 88, 173903 (2002)

26. M. Miranda, T. Fordell, C. Arnold, A. L'Huillier, H. Crespo, Opt. Express 20, 688-697 (2012)

27. M. Miranda, T. Fordell, C. Arnold, F. Silva, B. Alonso, R. Weigand, A. L'Huillier, H. Crespo, Opt. Express 20, 18732-18743 (2012)
28. B. Alonso, I.J. Sola, O. Varela, J. Hernández-Toro, C. Méndez, J. San Román, A. Zaïr, L. Roso, J. Opt. Soc. Am. B 27, 933-940 (2010)

29. B. Alonso, M. Miranda, I.J. Sola, H. Crespo, Opt. Express 20, 17880-17893 (2012)

30. B. Alonso, R. Borrego-Varillas, I.J. Sola, O. Varela, A. Villamarín, M.V. Collados, J. San Román, J.M. Bueno, L. Roso, Opt. Lett. 36, 3867-3869 (2011)

31. V. Pervak, I. Ahmad, M.K. Trubetskov, A.V. Tikhonravov, F. Krausz, Opt. Express 17, 7943-7951 (2009)

32. J. Paye, IEEE J. Quantum Electron. 28, 2262-2273 (1992)

33. J. Paye, A. Migus, J. Opt. Soc. Am. B 12, 1480-1490 (1995)

34. B. Alonso, R. Borrego-Varillas, O. Mendoza-Yero, I.J. Sola, J. San Román, G. Mínguez-Vega, L. Roso, J. Opt. Soc. Am. B 29, 1993-2000 (2012)

35. E. Kim, H. Kim, J. Noh, J. Korean Phys. Soc. 46, 1342-1346 (2005) 